\title{
Air-displacement plethysmography for determining body composition in neonates: validation using live piglets
}

\author{
Anne Frondas-Chauty ${ }^{1,2}$, Isabelle Louveau ${ }^{3,4}$, Isabelle Le Huërou-Luron ${ }^{3,4}$, Jean-Christophe Rozé ${ }^{1,2}$, Dominique Darmaun²
}

INTRODUCTION: Air-displacement plethysmography (ADP) was developed as a noninvasive tool to assess body composition, i.e., the proportion of fat mass (\%FM) and lean body mass. The results of previous studies comparing ADP with labeled water dilution in infants and with chemical analysis in phantoms have validated the ADP approach indirectly. We assessed the precision and accuracy of measurements of \%FM proportions in live animals, using ADP in comparison with biochemical analyses.

METHODS: Three groups of 12 piglets each underwent four consecutive body composition assessments at 2, 7, and $21 \mathrm{~d}$ and were euthanized to determine whole-body lipid content by direct chemical analysis.

RESULTS: The average body weights were 1,490, 2,210, and $5,610 \mathrm{~g}$ at $\mathrm{d} 2, \mathrm{~d} 7$, and $\mathrm{d} 21$, respectively. The mean \%FM values determined by biochemical analysis and ADP were $8.63 \pm$ $4.08 \%$ and $8.01 \pm 4.03 \%$, respectively. Linear regression and Bland-Altman analyses indicated good agreement for \%FM. The root mean square coefficient of variation (RMS-CV) for ADP was $17.9 \%$, with a better precision in the higher fat mass range.

DISCUSSION: Despite its relatively poor precision in the low range of \%FM, ADP measures fat mass with reasonable precision and accuracy in the range of body weight encountered in low-birth-weight infants.

$\mathbf{T}$ he rate of growth in utero and in the first few months of postnatal life may be a determinant of the risk of developing metabolic disorders and obesity in adulthood (1). Epidemiological evidence suggests that, in full-term neonates, high protein intake during the first $2 \mathrm{y}$ of life may lead to excess fat mass accretion, correlating with obesity later in life (2). In extremely preterm infants, the velocity of growth during the first few weeks of life correlates with neurodevelopmental outcome: achieving maximal growth rate has therefore been a priority for neonatologists. However, weight gain has often been the sole parameter used to assess early growth, whereas evidence in the literature suggests that the composition of weight gain (i.e., fat mass vs. lean body mass) may be a key determinant of later metabolic outcome. There is therefore keen interest among neonatologists, pediatricians, nutritionists, and physiologists in arriving at an accurate determination of body fat mass and, more generally, body composition, so as to optimize the nutritional management of low-birth-weight infants.

Several techniques have been proposed to evaluate body composition (3). Although anthropometric indexes such BMI and skinfold thickness measurement have been used (4), they are not accurate and are not easy to perform in babies. Although dual-energy X-ray absorptiometry (DXA) (5) has been commonly used to assess body composition, it is not routinely performed at the bedside and is associated with a significant, albeit small, exposure to radiation $(8.9 \mu \mathrm{Sv}$ as compared with $20 \mu \mathrm{Sv}$ for a chest X-ray) (6). Therefore, DXA cannot be carried out repeatedly to monitor the time course of body composition in the same infant. Consequently, there is need for a safe technique that allows for multiple measurements. Recently airdisplacement plethysmography (ADP) has been developed to measure fat mass in young infants (7).

$\mathrm{ADP}$ is a noninvasive technique based on the measurement of body volume and using gas laws. However, the use of this method in babies has been described only in a very limited number of studies $(8,9)$. The available validation data are based on the use of bovine tissue phantoms (10). The latter method does not take into account two potentially confounding factors present in a living subject: breathing movements and the maintenance of body temperature (7). Moreover, assessment of body composition by ADP uses Fomon's reference for fat-free mass (FFM) density. The latter depends on the water content of lean body mass (FFM), which is highly variable during the first few months of life $(11,12)$. Any error in the assumed water content of FFM could therefore distort the results with respect to body composition. A proper method of validation of ADP is therefore required, involving the use of a live animal model. Given its size and physiology, the piglet has been commonly used as a model for human infants (13).

The aim of this study was to assess the accuracy and reproducibility of ADP in comparison with body chemical analyses in newborn piglets, used as a model for low-birth-weight human infants. 


\section{RESULTS}

\section{Range}

The piglets varied widely in weight $(1.03-8.49 \mathrm{~kg})$ and biochemical analysis of percent fat mass $\left(\% \mathrm{FM}_{\mathrm{BA}}\right)(3.2-16.4 \%)$ (Table 1). The ranges of body mass and body adiposity in the piglets covered the ranges encountered in preterm and term human babies between birth and 6 mo of age.

\section{Reproducibility}

In each piglet, we carried out four measurements. The coefficient of variation $(\mathrm{CV})$ values for the repeated measurements ranged between 2.2 and $44.1 \%$. The root mean square (RMS)-CV was $17.9 \%$. Table 2 reports $\mathrm{CV}$ values for each group of piglets. The values of RMS-CV and median CV for repeatedly measured piglets differed significantly depending on age ( $\mathrm{d} 2, \mathrm{~d} 7$, or $\mathrm{d} 21)$, with better precision in older piglets (RMS-CV: $26.7,11.6$, and $14.6 \%$ at $\mathrm{d} 2, \mathrm{~d} 7$, and $\mathrm{d} 21$, respectively). CV values also differed significantly between the three terciles of $\% \mathrm{FM}_{\mathrm{BA}}$, with less variability among piglets with a higher fat mass. The median $\mathrm{CV}$ was $19.3 \%$ (25th-75th percentiles: $13.4-29.1$ ) for $\% \mathrm{FM}<5.4 \%$, and $4.9 \%$ (3.8-9.3) for $\% \mathrm{FM}>10 \%$ (Figure 1). In linear regression analysis, $\mathrm{CV}$ was dependent only on $\% \mathrm{FM}_{\mathrm{BA}}\left(R^{2}\right.$ adjusted: 0.273$)$.

\section{Accuracy}

Mean \%FM values determined by biochemical analysis and ADP were $8.7 \pm 4.1 \%$ and $8.01 \pm 4.03 \%$, respectively. The mean difference $(-0.66 \pm 1.73 \%)$ was significantly different from

Table 1. Selected characteristics of the population of piglets studied and $\%$ fat mass determined by ADP

\begin{tabular}{lccccc}
\hline & $\begin{array}{c}\text { Number } \\
\text { of piglets }\end{array}$ & Minimum & Maximum & Mean & SD \\
\hline Length $(\mathrm{cm})$ & 34 & 42 & 75 & 55.8 & 10.3 \\
Weight $(\mathrm{kg})$ & 34 & 1.03 & 8.49 & 3.19 & 2.0 \\
\%FM, biochemical & 34 & 3.2 & 16.4 & 8.7 & 4.1 \\
\%FM, ADP & 34 & 1.7 & 15.8 & 8.0 & 4.0 \\
\hline
\end{tabular}

ADP, air-displacement plethysmography; \%FM, percentage of fat mass in percentage of body weight.

Table 2. Anthropometric measurements and \% fat mass (\%FM), determined by ADP and biochemical methods, in three groups of piglets measured at 2,7 , and $21 \mathrm{~d}$ of life

\begin{tabular}{lcccc}
\hline & Day 2 & Day 7 & Day 21 & $P$ \\
\hline Length $(\mathrm{cm})$ & $46 \pm 2.3$ & $51.7 \pm 4.2$ & $67.9 \pm 5.7$ & $<0.05$ \\
Weight $(\mathrm{kg})$ & $1.45 \pm 0.26$ & $2.21 \pm 0.55$ & $5.61 \pm 1.39$ & $<0.05$ \\
FM, & $4.1 \pm 0.5$ & $8.2 \pm 1.8$ & $13.0 \pm 2.7$ & $<0.05$ \\
biochemical & & & & \\
\%FM, ADP & $3.7 \pm 1.7$ & $7.9 \pm 2.2$ & $11.7 \pm 3.2$ & $<0.05$ \\
$\begin{array}{l}\text { Median CV } \\
\text { (25th-75th }\end{array}$ & $25.5 \%(14-32)$ & $9.8 \%(7-13)$ & $5.4 \%(4-10)$ & $<0.05$ \\
percentiles) & & & &
\end{tabular}

Data are presented as mean \pm SD ( $n=11-12$ animals per age group). Coefficient of variation $=100 \times \mathrm{SD} /$ mean .

ADP, air-displacement plethysmography; $\mathrm{CV}$, coefficient of variation; \% FM, percentage of fat mass in body composition. zero $(P=0.031)$. Linear regression and Bland-Altman analyses of \%FM obtained by each of the methods indicated good agreement (Figure 2). The regression analysis indicated a low standard error of the estimate (1.71) and a high $R^{2}(0.83)$.

As shown in Table 3, the correlation between $\% \mathrm{FM}_{\mathrm{BA}}$ and $\% \mathrm{FM}_{\mathrm{ADP}}$ persisted with $R^{2}>0.8$ when equations specific for the physiologic characteristics of piglets were incorporated into the software.

\section{DISCUSSION}

This study used live newborn piglets to compare the accuracy of body composition determination using ADP with that using biochemical analysis. A reasonably good agreement was observed between the results of ADP and biochemical analyses; ADP underestimated fat mass, the mean difference being $0.66 \%$ with an SD of $1.73 \%$. Even though the difference was statistically significant $(P=0.031)$, the difference seems small and may not be physiologically relevant. The agreement between the two methods was confirmed by the regression analysis of $\% \mathrm{FM}$, as per biochemical and ADP methods, and was not dependent on changes in \%FM. The findings agree with previous reports on the accuracy of ADP, with a better coefficient of determination $\left(R^{2}=0.83\right)$ in the current study than previously obtained with bovine tissue phantoms $\left(R^{2}=0.76\right)(9)$, and the comparison study with the four-compartment reference model using labeled water dilution in children $\left(R^{2}=0.73\right)(8)$. Such improved accuracy as compared with results from tissue phantoms (9) may be attributable to the use of ADP under conditions close to its recommended use, i.e., with live subjects breathing spontaneously and maintaining a stable body temperature (7). We anesthetized the piglets so as to facilitate the performance of the experiments. However, Ma et al. found that, in human infants, the level of activity of the infant had no effect on the value of fat mass measured (9). We therefore believe that sedation in the



Figure 1. Reproducibility of ADP determinations (expressed as withinsubject (V), based on four measurements performed on the same day in each individual piglet, as a function of fat mass. The population of piglets was divided into terciles of percentage fat mass (\%FM), as determined by a biochemical method. First tercile: $n=11$, median CV (\%FM, ADP) 19.3 (13.4-29.1), RMS-CV: $24.7 \%$. Second tercile: $n=11$, median CV (\%FM, ADP) 9.8 (6-17.5), RMS-CV: $13.1 \%$. Third tercile: $n=11$, median CV (\%FM, ADP) 4.9 (3.8-9.3), RMS-CV: $13.48 \%$. ADP, air-displacement plethysmography; $\mathrm{CV}$, coefficient of variation; RMS, root mean square. 

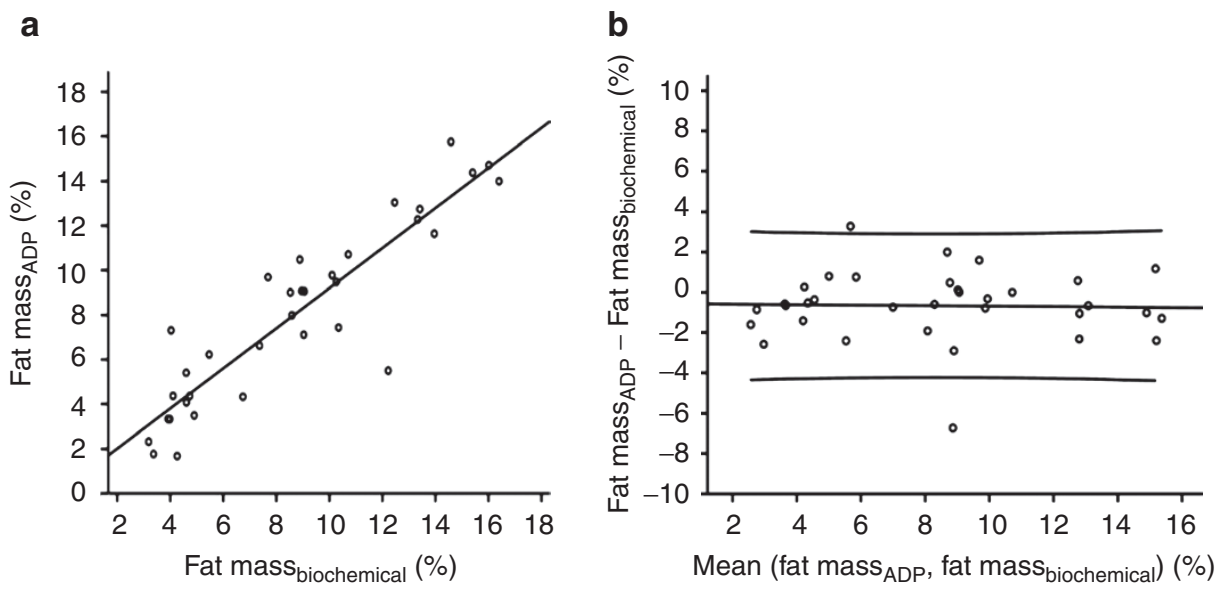

Figure 2. Comparison of percentage fat mass (\%FM) values determined by ADP and biochemical analyses of the carcasses. (a) Linear regression. Solid line: line for correlation, $r^{2}=0.83$. (b) Bland-Atman plot. Solid lines: 5 th and 95 th percentiles of the $\left(\% \mathrm{FM}_{\mathrm{ADP}}-\% \mathrm{FM}\right.$ biochemical analysis $)$ difference. ADP, airdisplacement plethysmography.

Table 3. Regression analysis of results of percentage fat mass (\%FM) determined using ADP and biochemical analysis, using various literature estimates of piglet fat mass density and various equations for the calculation of BSA

\begin{tabular}{|c|c|c|c|c|c|c|}
\hline \multirow{2}{*}{$\begin{array}{l}\text { Fat mass } \\
\text { density }\end{array}$} & ADP vs. carcass (\%) & \multirow[b]{2}{*}{ Regression slope } & \multirow[b]{2}{*}{$R^{2}$} & \multirow[b]{2}{*}{ SEE } & \multirow[b]{2}{*}{$P$ value } & \multirow[b]{2}{*}{ Reference } \\
\hline & Body surface area $\left(\mathrm{cm}^{2}\right)$ & & & & & \\
\hline 0.9007 & $\mathrm{BSA}=178.27 \mathrm{~L}(\mathrm{~cm})^{0.5} \cdot \mathrm{W}(\mathrm{kg})^{0.4838}$ & 0.909 & 0.827 & 1.705 & $<0.001$ & (26) \\
\hline 0.9140 & $\mathrm{BSA}=734 \mathrm{~W}(\mathrm{~kg})^{0.656}$ & 0.927 & 0.859 & 1.989 & $<0.001$ & (25) \\
\hline 0.9140 & $\mathrm{BSA}=1,210 \mathrm{~W}(\mathrm{~kg})^{0.575}$ & 0.905 & 0.819 & 1.813 & $<0.001$ & (23) \\
\hline
\end{tabular}

ADP, air-displacement plethysmography; BSA, body surface area; L, length (cm); SEE, standard error of the estimate; W, weight (kg).

piglets in our study was unlikely to have affected the validity of the ADP results. Ellis et al. (8) determined the accuracy of pediatric ADP measurement in comparison with indirect measurements of body composition using the four-compartment model in infants, whereas "true" \%FM, used as the reference value, was directly determined by biochemical analysis in our study.

We assessed the reproducibility of ADP determinations by carrying out four separate measurements of \%FM within less than $1 \mathrm{~h}$ in each piglet. The calculated RMS-CV between measurements is relatively high, at $17.9 \%$. Earlier studies of ADP variability in inanimate, non-animal phantoms, found measurements to be very precise (7). However, with animal phantoms, the $\mathrm{CV}$ was dependent on $\% \mathrm{FM}_{\mathrm{BA}}$, the $\mathrm{CV}$ being $18.4 \%$ with $\% \mathrm{FM}_{\mathrm{BA}} \leq 10 \%$ and $3.21 \%$ with $\% \mathrm{FM}_{\mathrm{BA}}>30 \%$ (10). In our study, the piglets were thin, with a mean $\% \mathrm{FM}_{\mathrm{BA}}$ of $8.7 \%$, and two-thirds of the population had $\mathrm{FM}_{\mathrm{BA}}$ of $<10 \%$. The mean CV was $8.6 \%$ for $\% \mathrm{FM}_{\mathrm{BA}}>10 \%$, which seems acceptable. Similarly, the clinical study by Ma et al. reported a change in CV of \%FM depending on body weight (9). Given that $\% \mathrm{FM}_{\mathrm{BA}}$ is often $>10 \%$ (14) in human infants, ADP using the Pea Pod is therefore suitable for the assessment of body composition in most infants. However, in small-for-gestational-age infants and preterm babies, given their lower body $\% \mathrm{FM}_{\mathrm{BA}}$, it may be prudent to repeat measurements at least twice, all the more so as the babies are able to move during the testing procedure (15).
Our study used a porcine model, which allows for the biochemical analysis of body composition. Previous studies by Ellis et al. (8), and Ma et al. (9) compared ADP results to measures of \%FM obtained through indirect methods $(8,9)$. The latter methods assess body composition on the basis of the measurement of total body water and may or may not be combined with the determination of bone mineral content and total body potassium. Both studies demonstrated the accuracy of the ADP method. Nevertheless, indirect measurements such as ADP assume that the density of FFM is known for each individual subject, despite the fact that total body water is known to change dramatically during the first 6 mo of life $(12,16)$. Such variations significantly alter the density of FFM $(11,12)$ and, consequently, the calculation of \%FFM and \%FM based on body density. Therefore, despite their obvious relevance, human validation studies are of limited value in assessing the accuracy of ADP. This was the rationale for our selection of biochemical analysis as the "gold standard" reference method, which obviously required an animal model.

Piglets have been used earlier to validate several techniques that are relevant for neonatal care (17-19), and are considered relevant models for the study of human nutrition $(13,20)$. The $\% \mathrm{FM}$ for a piglet is only $1 \%$ at birth, which corresponds to the $\% \mathrm{FM}$ of a preterm human infant with a gestational age of $30 \mathrm{wk}$. Early fat mass accretion is rapid in piglets; \%FM 
reaches up to $15 \%$ at $21 \mathrm{~d}$ of age, which is close to the $\% \mathrm{FM}$ of a human full-term newborn infant, or of a premature infant at the time of discharge from the neonatology unit $(13-15,21)$. In this study, the piglets selected covered a wide range of \%FM. Also, we used modified equations in the software formulas for \%FM so as to adapt them to porcine physiologic features (22-25). The fat mass density in piglets differs slightly from that in human infants (0.914 vs. 0.9007) (26). FFM density was assumed to be approximately the same in piglets and infants, taking into account the fact that total body water is identical between the two species (20). The software of the ADP instrument determines $\% \mathrm{FM}_{\text {adp }}$ on the basis of air pressure measurements in the test chamber and on anthropometric measurements including lung capacity and body surface area (7). Tidal volume and functional residual capacity are comparable in piglets and human infants; we therefore did not adjust the corresponding formulas in the software $(27,28)$. The modified formulas appropriate for the calculation of body surface area in piglets are described (22-25). The use of such modified equations did not result in significant changes in the accuracy of $\% \mathrm{FM}_{\text {adp }}$; for example, the coefficient of determination between ADP results and modification of fat mass density and body surface area (Deroth formula) (24) was 0.985 . We can therefore conclude that, in our study, ADP produced valid results in piglets.

$\mathrm{ADP}$ is of potential interest for the routine determination of body composition of infants in clinical practice. Anthropometry was found to lack accuracy (4). A comparison of the measurement of \%FFM and \%FM using DXA and biochemical analysis found DXA to be accurate for the determination of \%FFM but not for $\% \mathrm{FM}\left(R^{2}=0.36\right)$, with a $15 \%$ overestimation in the latter (19). In addition, the radiation associated with the measurement precludes the performance of multiple DXA scans for the monitoring of infant growth (5). Quantitative nuclear magnetic resonance was found to be accurate, based on a comparison with biochemical analysis in pigs, and was precise for body weights between 3 and $50 \mathrm{~kg}\left(R^{2}=0.87\right)(19)$. Although quantitative nuclear magnetic resonance is a quick and noninvasive technique just as ADP is, ADP has the additional advantage of being feasible in neonatal intensive care units because of the relatively small size and better mobility of the instrument and the shorter duration required to carry out the measurements.

In conclusion, Although ADP had previously been validated for the assessment of body composition in infants weighing $>2 \mathrm{~kg}$ or in inanimate tissue phantoms, this study is the first to compare ADP against the reference method, namely, biochemical analysis, in live, spontaneously breathing animals in the same weight range as preterm and low-birth-weight human infants. Although the precision of the ADP method was relatively poor in the low range of \% FM, ADP measured fat mass with reasonable precision and accuracy in live animals in the range of body weights seen in low-birth-weight human infants. Our findings suggest that pediatric ADP yields suitable accuracy and precision for use in clinical neonatal practice, provided repeated measurements are performed.

\section{METHODS}

\section{Animals}

The experiment was conducted in accordance with the legislations of the European Union (directive 86/609/CEE) and France (Décret: 2001$46429 / 05 / 01$ ) for the care and use of animals in research (agreement for animal housing number B-35-275-32 and certificate of authorization number 6061 to experiment on live animals). The scientific and technical staff obtained an agreement from the French veterinary service authorities to conduct animal research. To obtain a sample with a wide range of fat mass and body weight, we included three groups of 12 cross-bred piglets (Piétrain $\times($ Large White $\times$ Landrace $))$ from the experimental herd of INRA (St Gilles, France). Two piglets were selected per litter: one with a weight close to the mean litter birth weight $(1.30 \pm 0.03 \mathrm{~kg})$, and one with a birth weight of approximately the 10th percentile, corresponding to a birth weight of $0.90 \pm 0.02 \mathrm{~kg}$. Six pairs of piglets were separated from the respective sows at 2,7 , and $21 \mathrm{~d}$ of life. On the day of the experiment, if the weight was out of the range of ADP $(1-8 \mathrm{~kg})$, or if the piglet had died, another piglet within the same weight range was selected.

\section{Experimental Design}

The piglets were separated from the sows $2 \mathrm{~h}$ before they were scheduled to be killed. For $1 \mathrm{~h}$ before the test each piglet was housed in an individual cage or, if its weight was $<3 \mathrm{~kg}$, in an incubator (air temperature: $33^{\circ} \mathrm{C}$ ). General anesthesia was achieved with isoflurane $(1-8 \mathrm{vol} / 100 \mathrm{vol}$; C.S.P, Cournon d'Auvergne, France) delivered in $100 \%$ through a veterinary anesthesia ventilator. The piglets were not intubated, and they maintained spontaneous ventilation. Because the presence of excess hair would increase the volume of air trapped close to the skin, and could potentially alter ADP measurements, the piglets were shaved with clippers, washed, and dried. The umbilical cords of the younger piglets were cut before starting the experiment. Just before the first ADP test, an injection of $20 \mathrm{mg} / \mathrm{kg}$ of ketamine (Imalgene; Merial, Lyon, France) was administered into the trapezius muscle. Between each of the four ADP measurements, anesthesia with isoflurane was repeated. The piglets were killed at the end of the fourth ADP measurement, using anesthesia by isoflurane, followed by intracardiac T-61 injection ( $1 \mathrm{ml}$; Elvetis, Domloup, France). After death, the piglets were frozen until biochemical analysis.

\section{ADP System}

The Pea Pod (formerly Life Measurement, now COSMED, Rome, Italy) ADP infant body composition system is a new, noninvasive system designed to evaluate the body composition of babies weighing between 1 and $8 \mathrm{~kg}$. It determines the body volume of a baby placed in an airtight measuring chamber of $37 \mathrm{l}$. During the test, pressure variations are induced in the measuring chamber. The rise in pressure is linked to the air volume, by laws of gas compression, so that the determination of pressure changes allows determination of the baby's body volume. The ADP software calculates the baby's body density, on the basis of the body volume/weight ratio. The atmospheric air present in the lungs and the ambient air that remains trapped around the skin do not obey the same rules of compressibility; therefore, lung volume and body surface area must be taken into account in calculations. Because the presence of hair would increase the volume of air trapped close to the skin, thereby modifying the results of the test, the use of either a hair cap or oil to smooth down the hair has been recommended in human infants (7). In the case of the piglets, we decided to shave them. Body composition is considered as the sum of two compartments: fat mass and FFM. ADP estimates the density of FFM using values extrapolated for babies born preterm, on the basis of Fomon's and Butte's equations developed for full-term infants $(11,12)$, assuming the density of fat mass to be constant at 0.9007 . The ADP software then calculates the percentage of fat mass $\left(\% \mathrm{FM}_{\mathrm{ADP}}\right)$ on the basis of body density.

In order to use the instrument software designed for humans, we had to measure the "heights" of the piglets, and assign postnatal and gestational ages to the animals, because those parameters are required for the software to estimate body surface area and lung volume. Each piglet underwent four consecutive ADP tests. We measured the sizes 
of the piglets in terms of two parameters: (i) length from eyes to hind legs (EL) and (ii) length from snout to hind legs (SL). We used EL for the first and third ADP tests, and SL length for the second and fourth ADP tests. We then analyzed the impact of the method used for length measurement on Pea Pod determination of \%FM. The length measure from the snout improved the correlation between ADP values and those obtained from biochemical analysis $\left(r^{2}=0.844\right.$ vs. 0.787$)$. We therefore subsequently replaced in the Pea Pod's calculation spreadsheet the EL length used in first and third tests with the SL length to enable the Pea Pod software to produce two new values of \%FM using the SL length. For each piglet, we therefore had four values of \%FM using SL length, and two values of \%FM using EL length. The four results obtained using SL length allowed us to assess the reproducibility of the ADP.

Because the ADP software requires the baby's age, we also tried several approaches to enter the piglets' age into the software as if piglets were human babies. For the 21-d-old piglets, we entered the postnatal age of a human baby having the same weight as per French infant growth curves. For the 2- and 7-d-old piglets, we programmed the $\mathrm{ADP}$ as if the piglets were preterm human infants born the day of the test. If the anesthesia was not sufficient in any of the experiments, we immediately discontinued the measurement. The ADP software used pediatric formulas based on body surface area, functional residual lung capacity, and FFM density to calculate \%FM on the basis of pressure measurements $(7,11,12)$. Because the Pea Pod is designed for human infants, these correcting factors that are valid for humans are embedded in the software (7). We used piglet-specific equations from the veterinary literature to calculate body surface area in the piglets in our experiments (22-26), thereby obtaining appropriate \%FM values. We also entered the piglet-specific values of the density of fat mass into the APD software because the piglet-specific values differ from those in humans (0.9140 vs. 0.9007$)$.

\section{Chemical Analysis for Assessment of Body Composition}

Whole-body chemical analysis was performed on each piglet. The animals were stored at $-20^{\circ} \mathrm{C}$ immediately after killing. The frozen animals were minced, and samples were obtained and freeze-dried for further chemical analyses. Analyses were then performed in duplicate on freezedried samples. The residual water content after freeze-drying was determined from a 2.0 -g sample by drying at $+105^{\circ} \mathrm{C}$ to constant weight. Lipid content was determined from a 2.5-g sample by solvent extraction using an automatic Soxtec Avanti 2050 extraction system (FOSS, Höganäs, Sweden) as described previously (29). In brief, lipids were extracted using a chloroform and methanol mixture (chloroform:methanol, 2:1; Carlo Erba, Reuil-sur-Brêche, France). The CV (calculated as $(100 \times$ $\mathrm{SD}) /($ mean $)$ ) of the chemical lipid measurement was $7.4 \%$.

\section{Statistics}

All statistical analyses were performed using SPSS (Windows version 16.0; SPSS, Chicago, IL). The values are expressed as mean \pm SD. The reproducibility of $\% \mathrm{FM}_{\mathrm{ADP}}$ was determined by calculating the $\mathrm{SD}$ and $\mathrm{CV}=(100 \times \mathrm{SD}) /($ mean $)$ for four repeated measurements. Experimental reproducibility was evaluated using RMS-CV for the whole piglet population and for each population studied, as follows: RMS-CV: $\sqrt{ } \Sigma\left(\mathrm{CV}^{2} / n\right)$. For analyzing the differences in CV between piglets, we performed one-way ANOVA, comparing terciles of the population of piglets on the basis of fat mass and age. We also performed stepwise regression analysis, including body weight, body length, age, and \%FM as variables in the model.

In order to assess the accuracy of ADP measurements in comparison with results obtained by biochemical analyses, we performed a linear regression analysis with \%FM from biochemical analyses $\left(\% \mathrm{FM}_{\mathrm{BA}}\right)$ as the dependent variable. The coefficient of determination $\left(R^{2}\right)$ and the standard error of the estimate from the linear regression were also calculated. Bland-Altman analysis was used to determine the limits of agreement between the two methods as well as potential bias (30). A paired-sample $t$-test was used to detect any significant differences between $\% \mathrm{FM}_{\mathrm{ADP}}$ and $\% \mathrm{FM}_{\mathrm{BA}}$. In order to analyze the influence of our software adaptations (i.e., body surface area calculation, tidal volume, and fat mass density), we performed linear regression, analyzing the relation between $\% \mathrm{FM}_{\mathrm{ADP}}$ and $\% \mathrm{FM}_{\mathrm{BA}}$ for each hypothesis.

\section{ACKNOWLEDGMENTS}

We are grateful to Alessandro Urlando and Manoj Raghuraman for their generous help in adapting equations for application in piglets. They are employees of Life Measurement Inc. (now part of COSMED), the manufacturer of the Pea Pod Instrument. We also thank C. Tréfeu and G. Savary for their expert technical assistance. We are grateful to all the staff involved in the care of the animals.

\section{STATEMENT OF FINANCIAL SUPPORT}

This project was supported, in part, by grants from Agence Nationale de la Recherche (ANR-05-PNRA-009, Paris, France), the French Society of Neonatology, and Abbott Laboratories.

\section{REFERENCES}

1. Barker DJ. The developmental origins of adult disease. J Am Coll Nutr 2004;23:Suppl 6:588S-95S.

2. Rolland-Cachera MF, Deheeger M, Akrout M, Bellisle F. Influence of macronutrients on adiposity development: a follow up study of nutrition and growth from 10 months to 8 years of age. Int J Obes Relat Metab Disord 1995;19:573-8.

3. Ellis KJ. Evaluation of body composition in neonates and infants. Semin Fetal Neonatal Med 2007;12:87-91.

4. Koo WW, Walters JC, Hockman EM. Body composition in neonates: relationship between measured and derived anthropometry with dualenergy X-ray absorptiometry measurements. Pediatr Res 2004;56: 694-700.

5. Shypailo RJ, Butte NF, Ellis KJ. DXA: can it be used as a criterion reference for body fat measurements in children? Obesity (Silver Spring) 2008; 16:457-62.

6. Blake GM, Naeem M, Boutros M. Comparison of effective dose to children and adults from dual X-ray absorptiometry examinations. Bone 2006;38:935-42.

7. Urlando A, Dempster P, Aitkens S. A new air displacement plethysmograph for the measurement of body composition in infants. Pediatr Res 2003;53:486-92.

8. Ellis KJ, Yao M, Shypailo RJ, Urlando A, Wong WW, Heird WC. Bodycomposition assessment in infancy: air-displacement plethysmography compared with a reference 4-compartment model. Am J Clin Nutr 2007;85:90-5.

9. Ma G, Yao M, Liu Y, et al. Validation of a new pediatric air-displacement plethysmograph for assessing body composition in infants. Am J Clin Nutr 2004;79:653-60.

10. Sainz RD, Urlando A. Evaluation of a new pediatric air-displacement plethysmograph for body-composition assessment by means of chemical analysis of bovine tissue phantoms. Am J Clin Nutr 2003;77:364-70.

11. Fomon SJ, Haschke F, Ziegler EE, Nelson SE. Body composition of reference children from birth to age 10 years. Am J Clin Nutr 1982;35:Suppl 5:1169-75.

12. Butte NF, Hopkinson JM, Wong WW, Smith EO, Ellis KJ. Body composition during the first 2 years of life: an updated reference. Pediatr Res 2000;47:578-85.

13. Puiman P, Stoll B. Animal models to study neonatal nutrition in humans. Curr Opin Clin Nutr Metab Care 2008;11:601-6.

14. Hull HR, Dinger MK, Knehans AW, Thompson DM, Fields DA. Impact of maternal body mass index on neonate birthweight and body composition. Am J Obstet Gynecol 2008;198:416.e1-6.

15. Giannì ML, Roggero P, Taroni F, Liotto N, Piemontese P, Mosca F. Adiposity in small for gestational age preterm infants assessed at term equivalent age. Arch Dis Child Fetal Neonatal Ed 2009;94:F368-72.

16. Polonovski C, Voyer M, Chaumeil JC, Courpotin C. [Nutrition et renutrition en pratique pédiatrique]. Nutrition and Nutritional Rehabilitation in Clinical Pediatric Practice. Paris, France: Expansion Scientifique Française, 1992:1-735.

17. Koo WW, Hammami M, Hockman EM. Validation of bone mass and body composition measurements in small subjects with pencil beam dual energy X-ray absorptiometry. J Am Coll Nutr 2004;23:79-84.

18. Sheng HP, Adolph AL, Smith EO, Garza C. Body volume and fat-free mass determinations by acoustic plethysmography. Pediatr Res 1988;24:85-9. 
19. Mitchell AD. Validation of quantitative magnetic resonance body composition analysis for infants using piglet model. Pediatr Res 2011;69:330-5.

20. Shulman RJ. The piglet can be used to study the effects of parenteral and enteral nutrition on body composition. J Nutr 1993;123:Suppl 2:395-8.

21. Rudolph BC, Stahly TS, Cromwell GL. Estimation of body composition of neonatal pigs via deuterium oxide dilution: validation of technique. J Anim Sci 1988;66:53-61.

22. Kraybill HF, Goode ER, Robertson RS, Sloane HS. In vivo measurement of body fat and body water in swine. J Appl Physiol 1953;6:27-32.

23. Wachtel TL, McCahan GR Jr, Knox FS 3rd. Methods of preparing porcine skin for bioassay of thermal injury. Mil Med 1977;142:536-8.

24. DeRoth L, Bisaillon A. Determination of body surface area in neonatal swine. Lab Anim Sci 1979;29:249-50.

25. Kelley KW, Curtis SE, Marzan GT, Karara HM, Anderson CR. Body surface area of female swine. J Anim Sci 1973;36:927-30.
26. Guo SS, Chumlea WC. Tracking of body mass index in children in relation to overweight in adulthood. Am J Clin Nutr 1999;70:145S-148S.

27. Krause MF, Jäkel C, Haberstroh J, Schulte-Mönting J, Hoehn T. Functional residual capacity determines the effect of inhaled nitric oxide on intrapulmonary shunt and gas exchange in a piglet model of lung injury. Pediatr Crit Care Med 2001;2:82-7.

28. Proquitté H, Kusztrich A, Auwärter V, Pragst F, Wauer RR, Schmalisch G. Functional residual capacity measurement by heptafluoropropane in ventilated newborn lungs: in vitro and in vivo validation. Crit Care Med 2006;34:1789-95.

29. Kloareg M, Noblet J, van Milgen J. Deposition of dietary fatty acids, de novo synthesis and anatomical partitioning of fatty acids in finishing pigs. Br J Nutr 2007;97:35-44.

30. Bland JM, Altman DG. Statistical methods for assessing agreement between two methods of clinical measurement. Lancet 1986;1:307-10. 